\title{
Generation of a new Gateway-compatible inducible lentiviral vector platform allowing easy derivation of co-transduced cells
}

\author{
Philippe De Groote ${ }^{1,2}$, Sasker Grootjans ${ }^{1,2}$, Saskia Lippens ${ }^{1,2}$, Chantal Eichperger ${ }^{1,2}$, Kirsten \\ Leurs $^{1,2}$, Irene Kahr ${ }^{1,2}$, Giel Tanghe ${ }^{1,2}$, Inge Bruggeman ${ }^{1,2}$, Wouter De Schamphelaire ${ }^{3}$, Corinne \\ Urwyler $^{1,2}$, Peter Vandenabeele ${ }^{1,2}$, Jurgen Haustraete ${ }^{1,2, \star}$, and Wim Declercq ${ }^{1,2, *}$ \\ ${ }^{1}$ Inflammation Research Center, the Flanders Institute for Biotechnology (VIB), Ghent, Belgium, \\ 2Department of Biomedical Molecular Biology, Ghent University, Ghent, Belgium, and Department \\ of Biomedical Molecular Biology, BCCM/LMBP Plasmid Collection, Ghent University, Belgium
}

*These authors share senior authorship.

BioTechniques 60:252-259 (May 2016) doi 10.2144/000114417

Keywords: lentiviral vectors; co-inducible expression; Tet-On; Gateway

Supplementary material for this article is available at www.BioTechniques.com/article/114417.

In contrast to most common gene delivery techniques, lentiviral vectors allow targeting of almost any mammalian cell type, even non-dividing cells, and they stably integrate in the genome. Therefore, these vectors are a very powerful tool for biomedical research. Here we report the generation of a versatile new set of 22 lentiviral vectors with broad applicability in multiple research areas. In contrast to previous systems, our platform provides a choice between constitutive and/or conditional expression and six different C-terminal fusions. Furthermore, two compatible selection markers enable the easy derivation of stable cell lines co-expressing differently tagged transgenes in a constitutive or inducible manner. We show that all of the vector features are functional and that they contribute to transgene overexpression in proof-of-principle experiments.

Lentiviral transduction is a potent tool to efficiently derive cell populations with stable transgene expression (1) or stable knockdowns using RNAi technology (2). Unlike other methods of gene transfer, lentiviral particles can transduce almost any cell type, even non-dividing cells (1). Lentiviral vectors are also used for scalable production of recombinant proteins because they easily allow the generation of cell populations stably overexpressing the target protein (3). Derivation of stable cell populations for biomedical research is often hampered by the intrinsic properties of the studied gene. For example, genes that induce cell death or compromise cellular adhesion, possibly leading to anoikis, cannot be overexpressed constitutively without compromising further subculturing of the cells. Furthermore, interpretation of results after long-term continuous gene overexpression or knockdown can be complicated by the activation of compensatory mechanisms (4) or by counter-selection of transgene-expressing cells. To overcome these issues, much effort has been expended in generating inducible expression systems (5-7), which also allow for complete isogenicity between the control condition and the induced condition. When using toxic genes, the selection or sorting of transduced cells also needs to be independent of gene induction.

Inducible tetracycline-responsive lentiviral expression vectors often do not include the genes for required trans-activators or repressors (7-9); therefore, they must be used with cell lines engineered to contain those regulatory genes. To circumvent the latter requirement, others have designed single-vector systems in which the regulatory genes are included within the same vector $(6,10)$. However, these platforms are either limited to inducible vectors only or they cannot easily create cells that co-express differently tagged transgenes, whether inducible, constitutive, or a combination of both. Indeed, this combinatorial potential would allow a plethora of experiments studying the relationship between two genes to be performed in almost any cell type. To address these issues, we have built a new lentiviral vector platform based on Invitrogen's pLenti6 vector backbone.

\section{Materials and methods \\ Vector generation}

For cloning of the different tag sequences, synthetic oligonucleotides (Supplementary Table S1) were mixed in equimolar concen-

\section{METHOD SUMMARY}

We generated a new versatile set of 22 Gateway-compatible lentiviral vectors allowing for constitutive and/or doxycycline-inducible protein expression. The vector set is designed to express proteins fused to fluorescent or non-fluorescent $\mathrm{C}$-terminal tags. 
trations, heat denatured, and annealed by gradual cooling to room temperature (RT). The oligos were designed such that Apal and Mlul compatible ends were generated after annealing. The resulting oligo-duplexes were consequently ligated into an Apal and Mlul digested pLenti6/V5-DEST vector (Invitrogen, Merelbeke, Belgium) using T4 DNA ligase (Fermentas, Thermo Fisher Scientific, Ghent, Belgium). Enhanced GFP (eGFP) and tdTomato sequences were amplified from the vectors pEGFP-C1 and ptdTomato (both purchased from Clontech, Saint-Germainen-Laye, France), respectively, using primers 5'-CGCTCGAGTATGGTGAGCAAGGGCG-3' and 5'-GCGGGCCCTCCTTGTACAGCTCGTC-3' and then cloned into the Xhol and Apal sites of the pLenti6 backbone in order to generate $\mathrm{C}$-terminal fusion proteins after Gateway-mediated recombination.

The puromycin resistance gene was PCR amplified from the pTRIPZ vector (Open Biosystems, GE Healthcare, Diegem, Belgium) using primers 5'-GGATCTGATCAGCACGTGACCATGGCCACCGAGTACAAGC-3' and 5'-CTCGAATTGTGCTTAGCTCAGGCACCGGGCTTGCG-3’ and then cloned into Pm/l- and B/pl-digested and tag-modified versions of the pLenti6 backbone using the CloneEZ PCR cloning kit (GenScript, Piscataway, NJ). For conditional expression, the TRE-CMV min $_{\text {promotor }}$ was PCR amplified from the PTRIPZ vector using the primers 5'-ATCTCGACGGTATCGATGGTCCGAGGTTCTAGACG-3' and 5'-ACCACACTGGACTAGTGACCGGTGCGATCTGAC-3' and then cloned into Clal- and Spel-digested and tag-modified versions of the pLenti6 backbone containing the blasticidin resistance gene (Blast ${ }^{\mathrm{R}}$ ) using the CloneEZ PCR cloning kit. To create an all-in-one inducible vector system, a reverse Tet transactivator 3 (rtTA3)-internal ribosome entry site (IRES)-puromycin resistance gene (puro $^{R}$ ) sequence (rtTA3-IRES-puro ${ }^{R}$ ) was subsequently cloned using the CloneEZPCR cloning kit into the latter vectors (containing

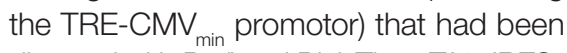
digested with Pm/l and B/pl. The rtTA3 -IRESpuro $^{R}$ sequence was PCR amplified from the pTRIPZ vector using the primers 5'-GGATCTGATCAGCACGTGACCATGTCTAGGCTGGACAAGAG-3' and 5'- CTCGAATTGTGCTTAGCTCAGGCACCGGGCTTGCG-3'. The resulting set of lentiviral vectors (Figure 1 and Supplementary Table S2) is publicly available through the BCCM/LMBP collection (www. bccm.belspo.be/). Vector sequences are also available through the EMBL Nucleotide
Sequence Database/European Nucleotide Archive (www.ebi.ac.uk/ena/data/view/ LT009438-LT009459). For expression and subsequent purification of murine soluble ST2 (msST2), transient transfection was performed with a pEF-msST2-mycHis expression plasmid (a gift from Harald Braun, Inflammation Research Center, VIB-UGent, Ghent, Belgium). For lentiviral transduction of a eukaryotic expression vector expressing msST2-mychis, cDNA was PCR amplified from the pEF-msST2-mycHis plasmid together with its natural secretion signal using the primers 5'- TTTACTAGTACCATGATTGACAGACAGAGA-3' and 5'- AAAACGCGTTCAATGGTGATGGTGATGATG-3'. The resulting amplicons, pLenti6-V5-puro and pLenti6-V5-blast were digested with Spel and Mlul and subsequently ligated using T4 DNA ligase (Fermentas), yielding the lentiviral expression plasmids pLenti6 msST2-mycHis puro and pLenti6 msST2-mycHis blast.

Cell culture and lentiviral transduction The HEK293T and keratinocyte HaCaT cell lines $(11,12)$ were cultured in DMEM supplemented with $10 \%$ FCS, $100 \mathrm{lU} / \mathrm{mL}$ penicillin, $0.1 \mathrm{mg} / \mathrm{mL}$ streptomycin, $0.03 \%$ L-glutamine, and $0.4 \mathrm{mM}$ sodium pyruvate (all purchased from Gibco, Thermo Fisher Scientific, Ghent, Belgium). For lentivirus production, HEK293T cells were transiently co-transfected with the indicated pLenti6 variants and packaging plasmids (pMD2-VSV-G and pCMV delta R8.91) using standard $\mathrm{CaPO}_{4}$ transfection. In brief, for each lentivirus production, $2.8 \times$ $10^{6}$ HEK293T cells were plated in a $75 \mathrm{~cm}^{2}$ tissue culture flask (Falcon, Thermo Fisher Scientific, Ghent, Belgium). The next day, the cells were transfected. Twelve micrograms lentiviral vector, 3.6 $\mu \mathrm{g}$ pMD2-VSV-G, and 7.8 ug pCMV delta R8.91 were added to a 1.5 $\mathrm{mL}$ final volume in $\mathrm{CaCl}_{2} / \mathrm{HEPES}$ buffer (25 $\mathrm{mM}$ HEPES, $\mathrm{pH}$ 7.05, $250 \mathrm{mM} \mathrm{CaCl}$ ). The latter solution was added dropwise to 1.5 $\mathrm{mL} 2 \times$ buffered saline (BS) $/ 1 \times$ HEPES buffer (25 mM HEPES, pH 7.05, 274 mM NaCl, 10 $\mathrm{mM} \mathrm{KCl}, 1.24 \mathrm{mM} \mathrm{Na}_{2} \mathrm{HPO}_{4}$, and $11 \mathrm{mM}$ dextrose) while vortexing. The solution was incubated for $1 \mathrm{~min}$ and added dropwise to the cells. After $6 \mathrm{~h}$, the medium was replaced, and the cells were incubated for another $48 \mathrm{~h}$ to allow for lentivirus production. Next, viruscontaining supernatant was harvested and filtered using a $0.45 \mu \mathrm{m}$ Millex-HV PVDF filter (Merck Millipore, Overijse, Belgium). Target cells were transduced by 2 consecutive infections for 8 h each with the obtained supernatants. Forty-eight hours after infection, stably transduced cells were selected as indicated. Upon completion of the antibiotic treatment in lentiviral transduced cultures, the remaining viable cell population was considered to be nearly $100 \%$ transduced based on complete cell death observed in non-transduced control cell cultures.Antibiotic selection was omitted during subsequent assays. Cells were plated in 6-well culture plates (Falcon) at 40,000 cells/well. Twenty-four hours later, transgene expression was induced by the addition of doxycycline (Dox) (Sigma-Aldrich, Diegem, Belgium). Phase contrast and fluorescence micrographs were taken on an Olympus IMT 200 microscope equipped with an AxioCam Mrm camera (Zeiss, Zaventem, Belgium). Images were contrast enhanced using AxioVision 4.8 software (Zeiss). Differential interference contrast (DIC) and confocal images were taken using a Zeiss observer Z1 spinning disk microscope. Lysates were prepared for Western blotting (WB) analysis using Laemmli buffer.

\section{Western blotting}

For WB analysis, $4.5 \mu \mathrm{L}$ culture supernatant was separated by SDS-PAGE and transferred to nitrocellulose. Protein lysates were prepared using colorless Laemmli buffer. Sample concentrations were determined by measuring absorbance at $280 \mathrm{~nm}$, and equal amounts of lysates were separated by SDS-PAGE, transferred to nitrocellulose, and analyzed by WB. Proteins were detected using the following antibodies: anti-His ${ }_{6}$ (penta His antibody; Qiagen, Venlo, The Netherlands), anti-Flag M2 (Sigma-Aldrich), anti-VSV-G (Sigma-Aldrich), anti-HA (BAbCO, Richmond, CA), anti-GFP Living Colors JL-8 (BD Biosciences, Erembodegem, Belgium), anti-Ecadherin clone 36 (BD Biosciences) and anti-b-tubulin-HRP (Abcam, Cambridge, UK).

Protein production and purification For the production of each recombinant protein, three $175 \mathrm{~cm}^{2}$ culture flasks (Falcon) were plated at $4 \times 10^{6} \mathrm{cell}$ s per flask in DMEM supplemented with $10 \%$ FCS, $100 \mathrm{IU} / \mathrm{mL}$ penicillin, $0.1 \mathrm{mg} / \mathrm{mL}$ streptomycin, $0.03 \%$ L-glutamine, and $0.4 \mathrm{mM}$ sodium pyruvate (all purchased from Gibco). For transient transfection, an identical number of untransduced HEK293T cells or stably transduced HEK293T cells were plated $\left(4 \times 10^{6}\right.$ cells per $175 \mathrm{~cm}^{2}$ flask). Twenty-four hours after plating, cells were transiently transfected or not using standard $\mathrm{CaPO}_{4}$ transfection. Forty-eight hours after plating, the medium was switched to serum-free DMEM supple- 
A

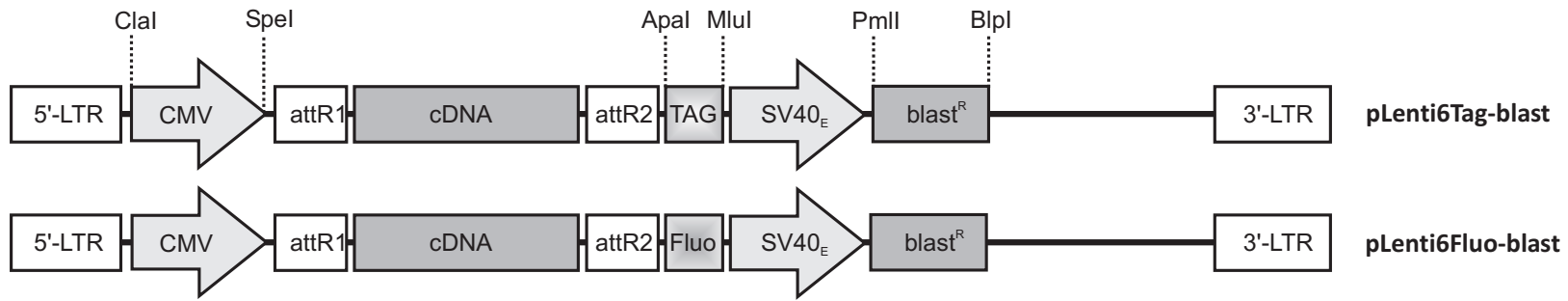

B
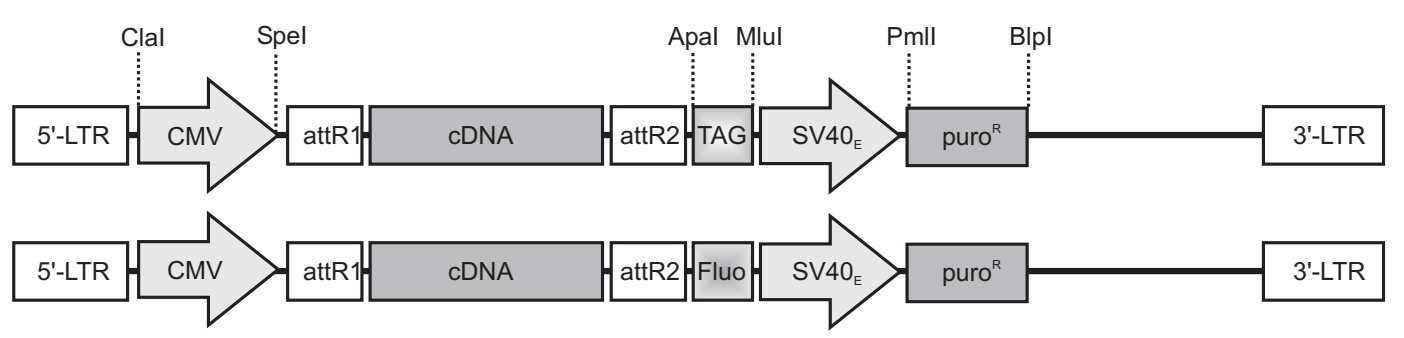

pLenti6Tag-puro

C
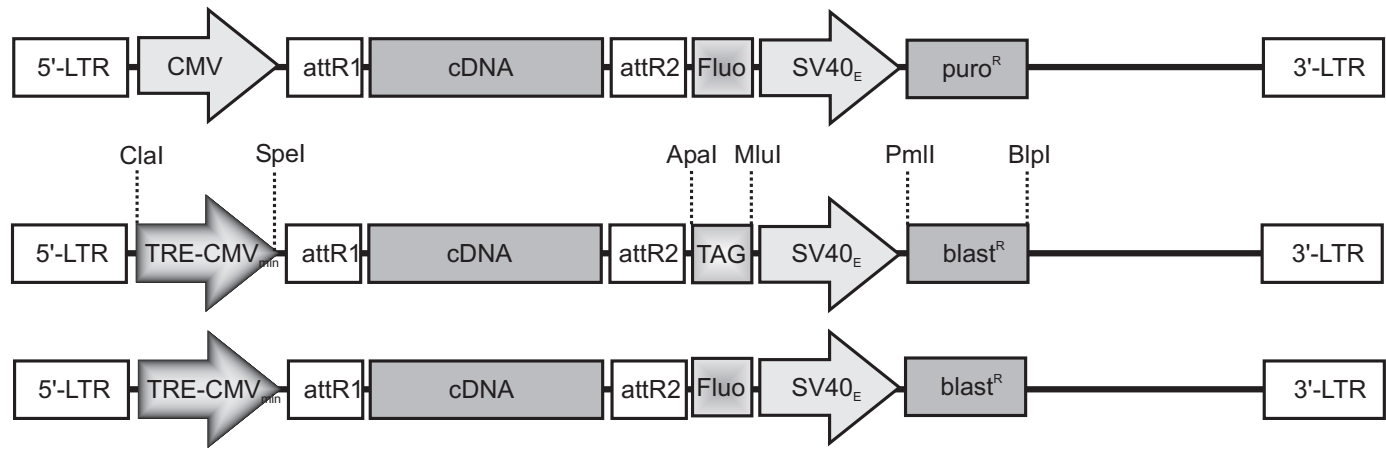

pLenti6Fluo-puro

D
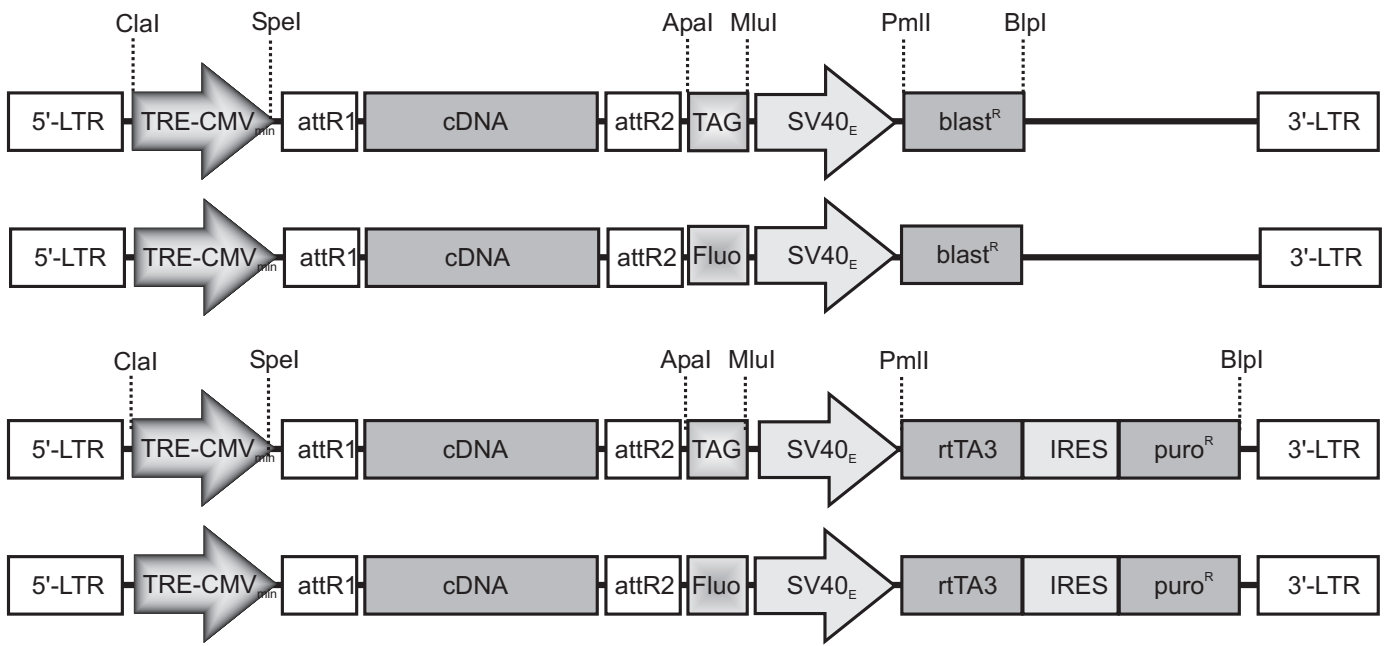

pDG1iTag-blast

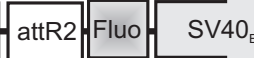

$40_{\mathrm{E}}>$ blast $^{\mathrm{R}}$

3'-LTR

pDG1iFluo-blast

E
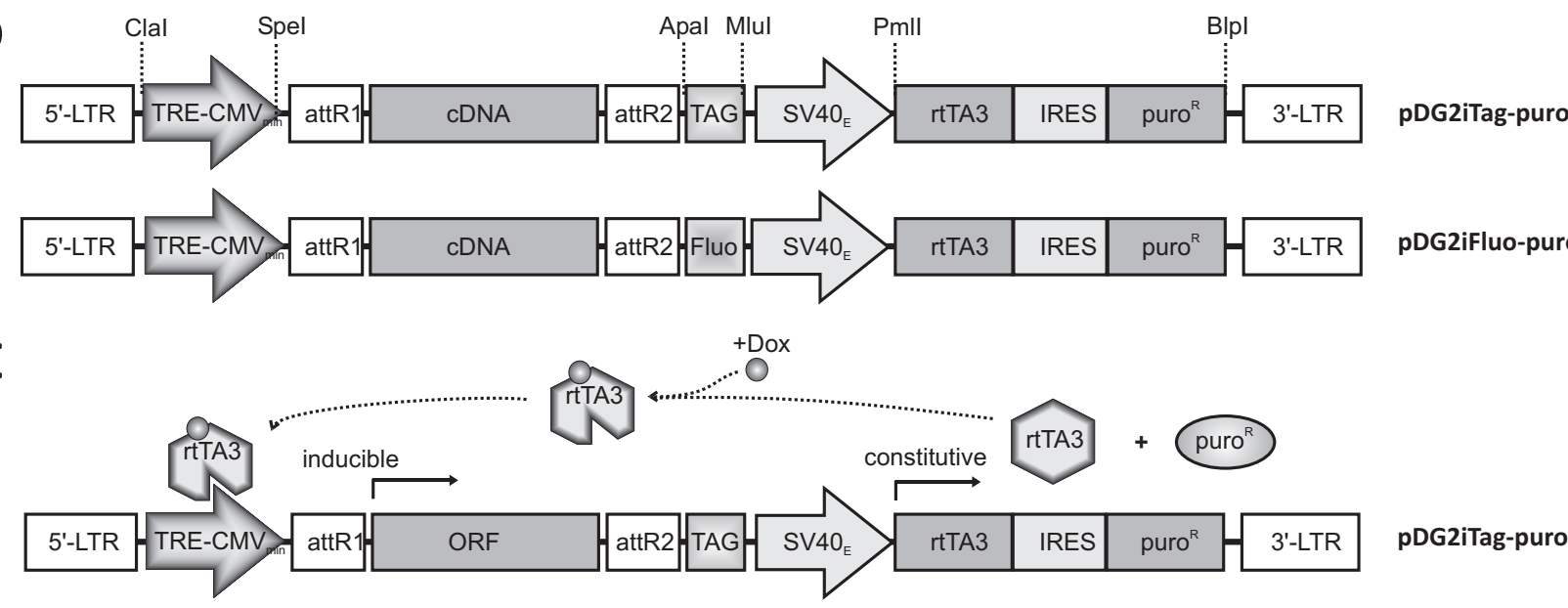

pDG2iFluo-puro

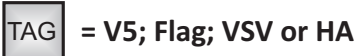

Fluo = tdTomatoRed-V5 or eGFP-V5

Figure 1. Overview of the lentiviral vector platform. (A) Diagrams of the constitutive, blasticidin-selectable vectors. (B) Diagrams of the constitutive, puromycin-selectable vectors. (C) Diagrams of the inducible, blasticidin-selectable vectors. (D) Diagrams of the all-in-one inducible, puromycin-selectable vectors. (E) Diagram showing the principle of the all-in-one inducible lentiviral expression vector design. (A-D) For each vector type, variants are available that allow C-terminal fusion of either V5-, Flag-, VSV-, or HA epitopes or tdTomatoRed-V5 or eGFP-V5 fluorescent proteins.

mented with insulin-transferrin-selenium (ITS) at a final concentration of $10 \mathrm{mg} / \mathrm{L}$ insulin, 5.5 $\mathrm{mg} / \mathrm{L}$ transferrin, and $0.0067 \mathrm{mg} / \mathrm{L}$ sodium selenite (Catalog \#41400-045; Gibco), 2 $\mathrm{g} / \mathrm{L}$ Peptone Primatone RL (Sigma-Aldrich), $0.03 \%$ L-glutamine (Gibco), and $0.4 \mathrm{mM}$ sodium pyruvate (Gibco). Every $72 \mathrm{~h}$, the culture supernatant was collected and pooled separately for each condition. Cell supernatant was centrifuged $(16700 \times \mathrm{g}, 30 \mathrm{~min}$, $4^{\circ} \mathrm{C}$ ) in a Fiberlite F10-5x500y rotor (Thermo
Fisher Scientific, Ghent, Belgium) and passed through a $0.22 \mu \mathrm{m}$ GP Millipore Express bottle top filter (Merck Millipore). Subsequently, the sample was dialyzed against sample buffer $\left(20 \mathrm{mM} \mathrm{NaH}_{2} \mathrm{PO}_{4}, \mathrm{pH} 7.4,500 \mathrm{mM} \mathrm{NaCl}\right.$, $20 \mathrm{mM}$ imidazole) and loaded on a $1 \mathrm{~mL}$ HisTrapTM HP column (GE Healthcare) that was equilibrated with sample buffer. The purification was performed using the Äkta explorer platform (GE Healthcare). After loading, the column was first washed using $25 \mathrm{~mL}$ wash buffer $1\left(20 \mathrm{mM} \mathrm{NaH}_{2} \mathrm{PO}_{4}, \mathrm{pH} 7.4,500 \mathrm{mM}\right.$ $\mathrm{NaCl}, 20 \mathrm{mM}$ imidazole, 0.1\% Empigen) and then with $15 \mathrm{~mL}$ wash buffer $2(20 \mathrm{mM}$ $\mathrm{NaH}_{2} \mathrm{PO}_{4}, \mathrm{pH} 7.4,500 \mathrm{mM} \mathrm{NaCl}, 50 \mathrm{mM}$ imidazole). The His-tagged protein was eluted in $0.5 \mathrm{~mL}$ fractions using $20 \mathrm{~mL}$ elution buffer (20 mM NaH $\mathrm{PO}_{4}, \mathrm{pH} 7.4,500 \mathrm{mM} \mathrm{NaCl}, 400$ $\mathrm{mM}$ imidazole). Twenty-five microliters of each fraction was separated by standard reducing SDS-PAGE and analyzed by Coomassie blue staining. The purified fractions were pooled 
based on the Coomassie-stained gels. For analysis of protein yield, samples from each pool were desalted against PBS using a Zeba 96-well spin desalting plate (Pierce, Thermo Fisher Scientific, Ghent, Belgium), and the protein concentration was measured using a standard micro BCA assay (Thermo Fisher Scientific, Ghent, Belgium).

\section{Results and discussion}

A series of lentiviral vectors was designed to fulfill the following requirements: (i) Gateway-compatibility for easy transfer of DNA sequences between different vectors; (ii) availability of both constitutive and inducible versions that can be combined; (iii) the regulatory trans-activator should be encoded on the same transfer vector as the inducible expression cassette to allow one-step transduction for inducible expression; (iv) use of different protein tags to allow selective immune reactivity toward the different transgenes without the need for protein-specific antibodies; ( $v$ ) fusion with fluorescent proteins to track subcellular localization of the expressed protein; (vi) different selection markers that allow rapid selection of co-transduced cells.
Specific detection or purification of overexpressed proteins without the need for protein-specific antibodies requires that tags be fused to the proteins. Therefore, we opted to provide multiple short immunereactive or fluorescent tags in our system. We adapted the original Gateway-compatible pLenti6 vector to allow a choice between four widely used immune-reactive tags (V5, Flag, VSV, or HA) and two fluorescent tags (eGFP or tdTomatoRed). If the fusion of the tag does not alter the biological functionality of the protein in question, then the fluorescent tags could be used to study the influence of gene knock-down or overexpression of one gene on the subcellular localization of a second gene, without the need for cumbersome staining, thus enabling highcontent screening.

Each of these tags are expressed as a C-terminal fusion to the transgene of interest (Figure 1A). In order to permit rapid selection of co-transduced cells, we provided our vector set with two different compatible selection markers: blasticidin (blast $\left.{ }^{R}\right)$ and puromycin $\left(\right.$ puro $\left.^{R}\right)$ resistance genes (Figure 1B). We also supplemented our system with a tetracycline (Tet)-responsive promoter originating from the pTRIPZ-vector (Open
Biosystems) that consists of a stretch of Tet-responsive elements (TRE) fused to a minimal CMV-promoter $\left(\mathrm{CMV}_{\text {min }}\right)$, leading to the pDG1iTag/Fluo-blast versions of our vector platform (Figure 1C). However, to provide a single vector system containing both the tetracycline regulatory transactivator and the inducible expression cassette, we also cloned a sequence encoding rtTA3IRES-puro ${ }^{R}$ under the control of the SV40 early promoter into our pDG1iTag/Fluoblast destination vectors to yield the all-inone inducible lentiviral expression vectors pDG2iTag-puro and pDG2iFluo-puro (Figure 1, $D$ and E). Transcription of the rtTA3-IRESpuro $^{R}$ sequence will lead to a bicistronic transcript encoding rtTA3 that improves Dox sensitivity and activity $(13,14)$, and the puro $^{R}$ selection marker (Figure 1E). In all, we have generated a set of 22 new vectors with a choice of 6 different C-terminal fusions, each with either blast ${ }^{R}$ or puro ${ }^{R}$ and either constitutive or Dox-inducible expression (Figure 1). The resulting set of lentiviral vectors (Figure 1 and Supplementary Table S2) is publicly available through the BCCM/LMBP collection (www.bccm.belspo.be/).

The use of lentiviral vectors for the derivation of stable cell populations producing

A

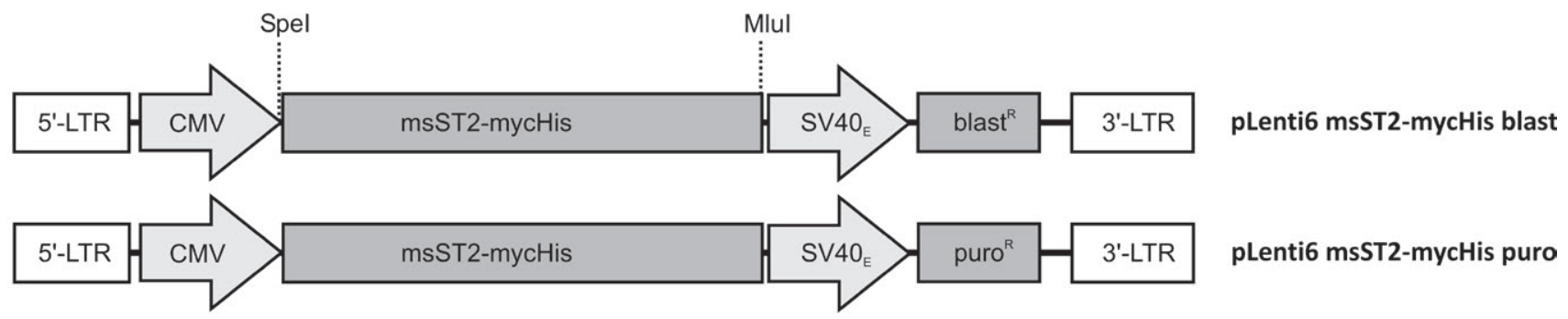

B

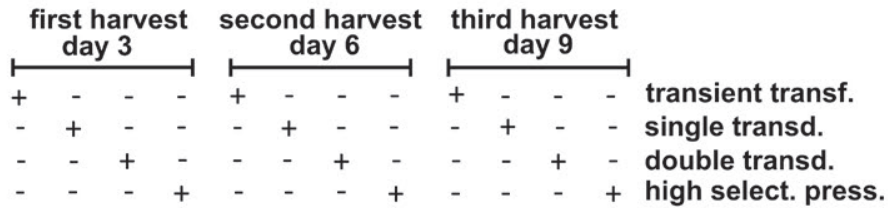
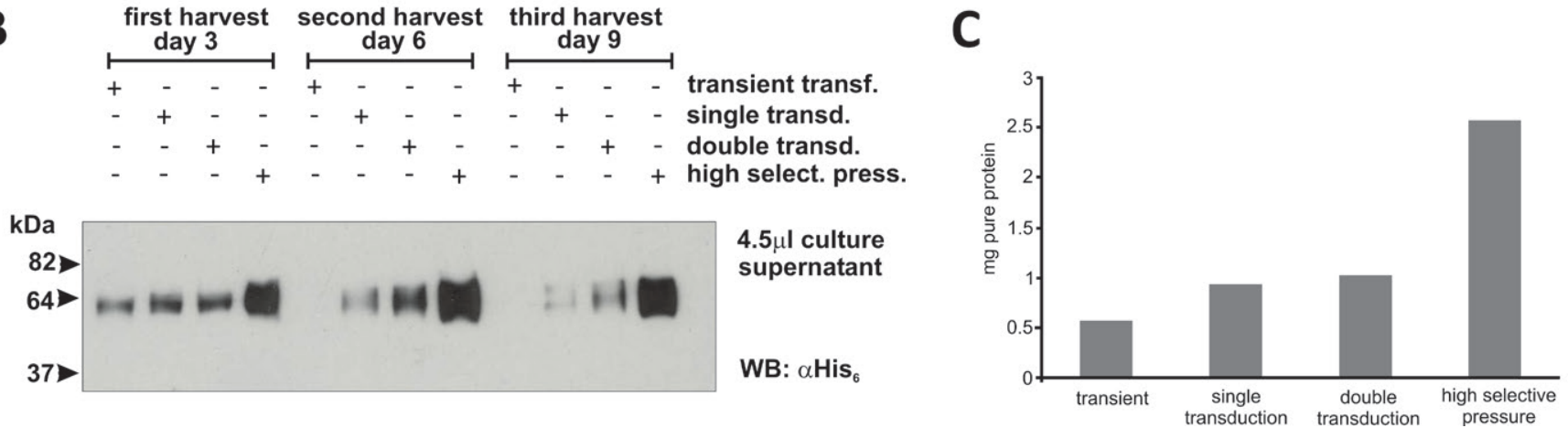

Figure 2. Easy derivation of stable cell populations with increased levels of recombinant protein expression. (A) Diagrams of the lentiviral expression constructs used for the generation of stable cell populations producing secreted recombinant msST2-mycHis. msST2-mycHis cDNA, including its natural secretion signal, was cloned between the Spel and M/ul restriction sites. (B) Anti-His ${ }_{6}$ Western blotting of culture supernatant from the indicated HEK293T cell populations expressing msST2-mycHis. HEK293T cells were transiently transfected (transient transf.) or transduced with pLenti6 msST2-mycHis puro and selected with $0.5 \mu \mathrm{mg} / \mathrm{mL}$ puromycin for $48 \mathrm{~h}$, yielding the single transduced cell population (single transd.). The latter population was superinfected with pLenti6 msST2-mycHis blast and selected for $48 \mathrm{~h}$ with $1 \mu \mathrm{g} / \mathrm{mL}$ blasticidin, yielding the double transduced cell population (double transd.). Finally, this double transduced population was subjected to increasing concentrations of both puromycin and blasticidin. A highly resistant cell population (high select. press.) was finally obtained by a $72 \mathrm{~h}$ selection using $3.0 \mu \mathrm{g} / \mathrm{mL}$ puromycin and $5.5 \mu \mathrm{g} / \mathrm{mL}$ blasticidin. For each condition, $90 \mathrm{~mL}$ culture supernatant was harvested from three $175 \mathrm{~cm}^{2} \mathrm{flasks}$ at days 3,6 , and 9 and analyzed by Western blotting as indicated. (C) Total pure msST2-mycHis yield after nickel affinity chromatography and dialysis. All $90 \mathrm{~mL}$ harvests were purified separately by nickel chromatography and dialyzed. Protein concentration was measured by micro BCA, and total pure protein yield was calculated for each cell population. The second and third harvests from the transiently transfected cells were omitted from the calculation due to their impurity (see also Supplementary Figure S1). 
A

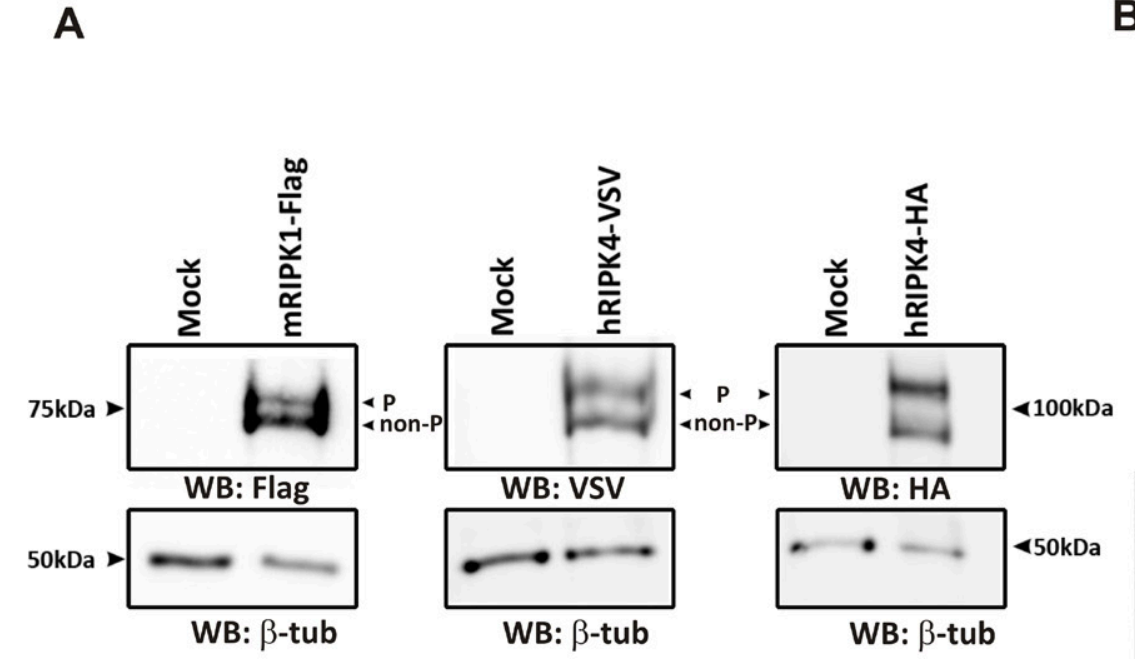

B

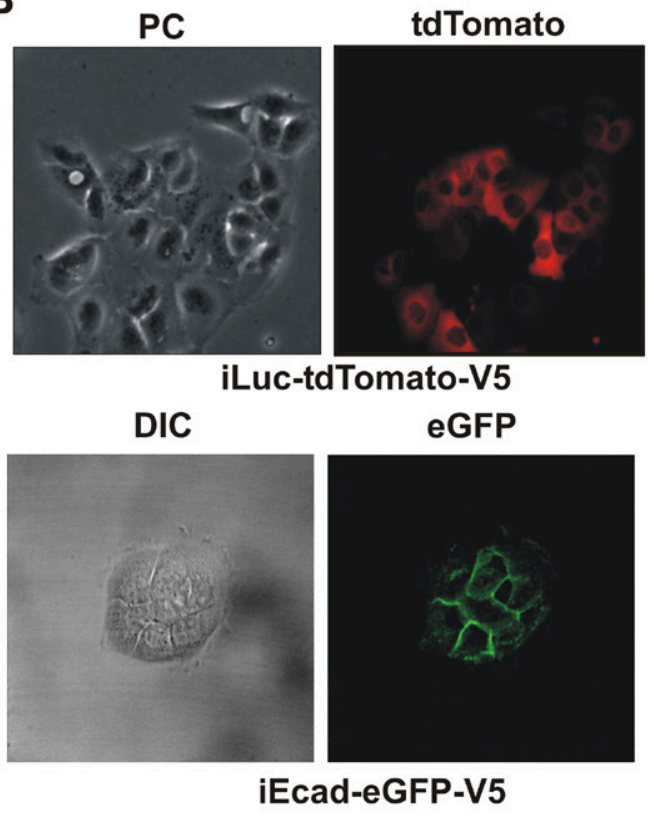

C

L929s

pLenti6 histone2-eGFP-V5-Puro

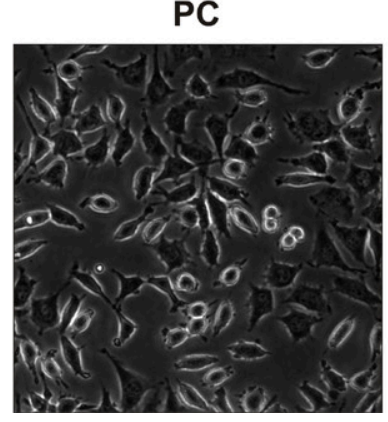

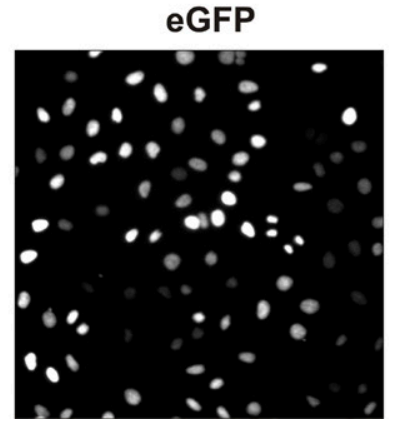

Composite

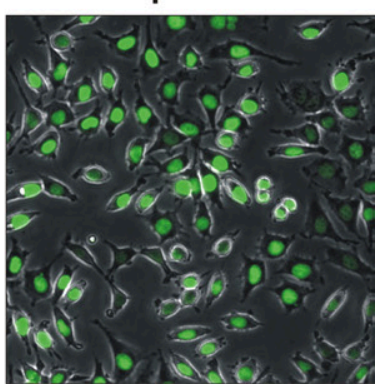

Figure 3. Functional characterization of the C-terminal fusions introduced into the pLenti6 backbone. (A) Western blotting of lysates from HEK293T cells transiently transfected with the indicated pLenti6-constructs. The overexpressed receptor interacting protein kinase (RIPK) proteins were detected as double bands, due to post-translational autophosphorylation. b-tubulin served as loading control. P, phosphorylated RIPK; non-P, non-phosphorylated RIPK. (B) Phase contrast and fluorescence microscopy analysis of $\mathrm{HaCaT}$ cells transduced with pDG2iLuc-tdTomatoRed-V 5 and induced for $24 \mathrm{~h}$ by addition of $1 \mu \mathrm{gg} / \mathrm{mL}$ doxycycline (Dox) (upper panels). Differential interference contrast (DIC) and confocal fluorescence microscopy analysis of HaCaT cells transduced with pDG2iLucFlag puro and pDG1iE-cad-eGFP-V5 blast and selected using $5 \mu \mathrm{g} / \mathrm{mL}$ blasticidin for $96 \mathrm{~h}$ and $2 \mu \mathrm{g} / \mathrm{mL}$ puromycin for $48 \mathrm{~h}$ (lower panels). Transgene expression was induced for $48 \mathrm{~h}$ by addition of $0.5 \mu \mathrm{g} / \mathrm{mL}$ Dox. (C) Phase contrast and fluorescence microscopy analysis of L929s cells transduced with the pLenti6 histone2-eGFP-V5-Puro construct and selected with $2 \mu \mathrm{g} / \mathrm{mL}$ puromycin. Note the nucleus-specific expression of enhanced GFP (eGFP)-tagged histone 2.

recombinant proteins was described earlier (3). We exploited the presence of the blasticidin or puromycin selection marker in our lentiviral expression vectors to easily increase recombinant protein expression by double transduction and selection for high copy numbers by increasing blasticidin and puromycin concentrations. Therefore, we compared the expression of myc/His tagged mouse sST2 (msST2-mycHis), a secreted soluble IL-33 receptor extracellular domain, from HEK293T cells that were: (i) transiently transfected; (ii) stably transduced with one or two selectable constructs; or (iii) doubletransduced and selected for high copy numbers (Figure 2A). After transient transfection or selection of the stable cell populations, culture supernatant was harvested every 3 days (days 3, 6, and 9), and msST2-
mycHis was IMAC-purified from each harvest separately. msST2-mycHis expression levels were compared based on WB of the culture supernatant (Figure 2B) and by comparison of the purified protein yield of the IMAC-purifications (Figure 2C and Supplementary Figure S1). HEK293T cells that were transiently transfected showed a rapid decrease in the level of secreted protein expressed within a 6-day time frame, whereas these levels decreased much less for each of the stably transduced polyclonal populations (Figure 2B and Supplementary Figure S1). For transiently transfected cells, the IMAC-purifications from the second and third harvests contained a substantial amount of contaminating protein (Supplementary Figure S1). Therefore the second and third harvests from the transiently transfected cells could not be considered as pure msST2-mycHis protein. Superinfected cells only showed a marginal increase in the expression of msST2-mycHis compared with cells transduced with only one construct. In contrast, the total protein yield of msST2-mycHis purified from the 3 consecutive harvests was increased 2.5-fold through selection of the double-transduced polyclonal population with high concentrations of both puromycin and blasticidin (3.0 $\mu \mathrm{g} / \mathrm{mL}$ and $5.5 \mu \mathrm{g} / \mathrm{mL}$, respectively) (Figure 2C). Co-expression of chaperones is often beneficial to the yield of recombinant bioactive protein $(15,16)$. By making use of the two selectable markers, our platform would also be perfectly suited for generating such stable cell lines.

To address the functionality of the introduced Flag-, VSV-, and HA-tags, we 
Figure 4. Co-transduction of pDG1i-vectors and pDG2i-vectors allows co-induction of different transgenes. (A) Scheme for the co-induction of two different transgenes upon co-transfection of the pDG1i- and pDG2i-vectors. Upon doxycycline (Dox) addition, the reverse Tet transactivator 3 gene (rtTA3) in the pDG2i-plasmid will bind and activate transcription from the Tet-responsive promoters of both constructs, leading to co-induction of both transgenes. (B) Upper panels represent phase contrast microscopy analysis of a control selection on untransduced cells. Blasticidin was used at 5 $\mu \mathrm{g} / \mathrm{mL}$ for $96 \mathrm{~h}$, while puromycin was used at $2 \mu \mathrm{g} /$ $\mathrm{mL}$ for $48 \mathrm{~h}$. Lower panels represent phase contrast microscopy analysis of $\mathrm{HaCaT}$ cells co-transduced with pDG1iEcad-eGFP-V5 blast and pDG2iLucFlag puro that were selected using $5 \mathrm{\mu g} / \mathrm{mL}$ blast for $96 \mathrm{~h}$ and $2 \mu \mathrm{g} / \mathrm{mL}$ puro for $48 \mathrm{~h}$, after which transgene expression was induced for $48 \mathrm{~h}$ using $1 \mathrm{\mu g} /$ $\mathrm{mL}$ Dox. (C) Western blotting (WB) directed against the $\mathrm{C}$-terminal fusions of the indicated transgenes. Cells from the lower panels in B were lysed and subjected to anti-Flag and anti-GFP immunoblotting. b-tubulin served as the loading control. (D) Time-course kinetic analysis of Dox-dependent transgene induction. Anti-Flag WB of lysates from $\mathrm{HaCaT}$ cells stably transduced with pDG2iRIPK4Flag puro, selected for $48 \mathrm{~h}$ using $2 \mu \mathrm{g} / \mathrm{mL}$ puromycin and induced for the indicated time by addition of $1 \mu \mathrm{g} / \mathrm{mL}$ Dox. b-tubulin immunoblotting served as the loading control. (E) Dose-dependent transgene induction by Dox titration. Transgene expression was induced with the indicated Dox concentration in the stably co-transduced $\mathrm{HaCaT}$ cells from (B). Transgene expression was detected by anti-Flag and anti-E-cadherin immunoblotting. b-tubulin served as the loading control.

performed $L R$ recombination of the constitutive vectors with an entry vector containing the cDNA for the human receptor interacting protein kinase 1 (RIPK1) or receptor interacting protein kinase 4 (RIPK4) genes. After transient transfection of the resulting vectors in HEK293T cells, we could detect protein expression by means of WB using antibodies directed against the specified protein tags (Figure 3A, upper panels). As expected based on the literature, both RIPK proteins were detected as double bands by WB due to the presence of non-phosphorylated and hyperphosphorylated forms of the protein $(17,18)$. The functionality of the tdTomato-V5 and eGFP-V5 fusions was examined by fluorescence microscopy. Epithelial $\mathrm{HaCaT}$ cells were transduced with inducible luciferase-tdTomato-V5 (pDG2iLuc-tdTomato-V5) or E-cadherineGFP-V5 (pDG1iEcad-eGFP-V5) and pDG2iLuc-FLAG-puro (see below) constructs and induced with Dox. Fluorescence microscopy showed that both fusion proteins were well-expressed and could be detected based on the red or green fluorescence of the respective fluorescent protein tags (Figure 3B). Similar to endogenous E-cadherin, E-cadherin-eGFP was mainly expressed at
A
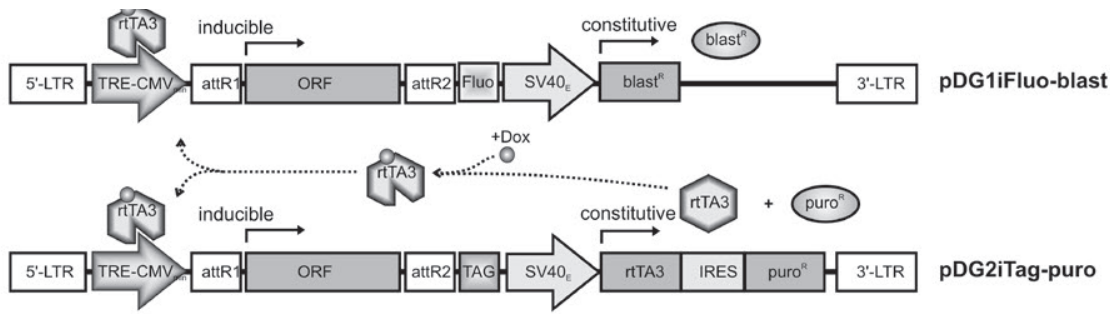

B
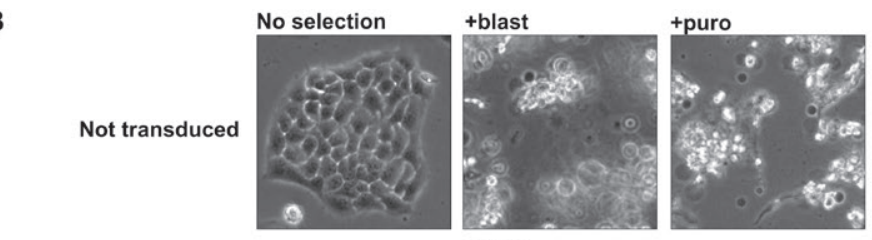

C

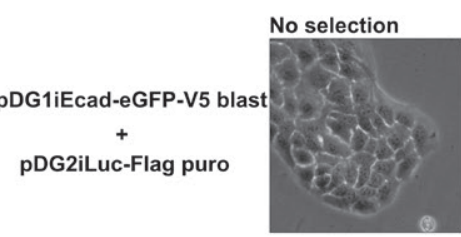

+puro
+blast

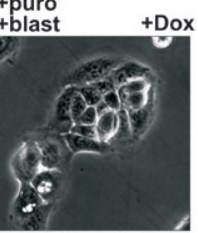

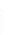

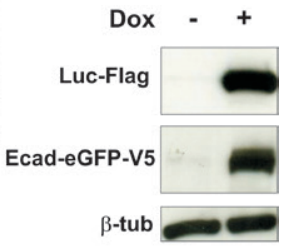

D

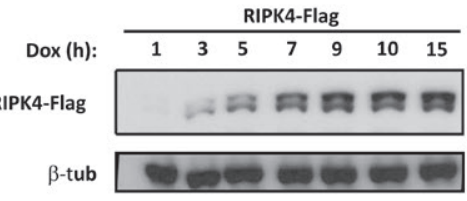

E

iLucif-Flag / iEcad-eGFP-V5

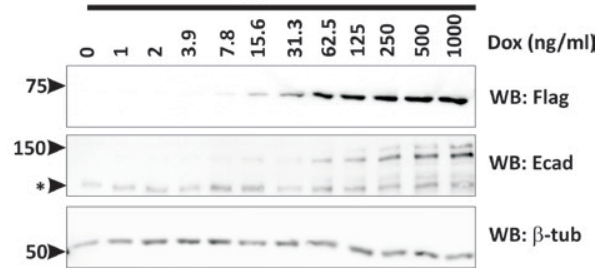

represents storage of excess E-cadherin in E-cadherin-eGFP-V5-positive vesicles. The heterogeneous expression levels seen in these polyclonal populations could also be exploited to select for cells with a desired uniform expression level by clonal selection or fluorescence assisted cell sorting (FACS) (Figure 3, B and C, and Supplementary Figure system to produce cells with stable nucleusspecific fluorescence (Figure 3C) or wholecell fluorescent marking (Supplementary Figure S2A) by expressing, respectively, eGFP-tagged histones or eGFP alone. Thus, this system is ideal for easily generating cell lines for time-lapse experiments S2). In addition, we also used our expression white arrowheads). The latter most likely addition, cells with the highest E-cadherineGFP levels also showed more pronounced punctuate intracellular E-cadherin-eGFP-V5 expression (Supplementary Figure S2B,

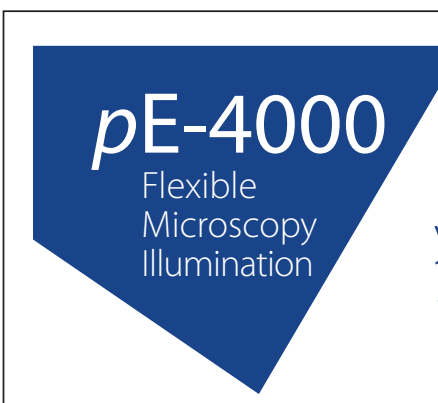

f于圆 in Every wavelength you'll ever need!

16 selectable wavelengths

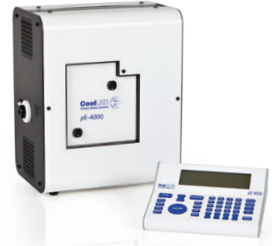

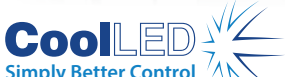

+44 (0)1264323040 (Worldwide) 1-800-877-0128 (USA/Canada) e: info@CoolLED.com www.CoolLED.com 
or screenings that involve changes in cell morphology or the subcellular localization of certain proteins. Note that, as reported for GFP (20), eGFP alone tends to show higher levels in the nucleus compared with the cytoplasm (Supplementary Figure S2A).

Combined expression of differently tagged proteins is often used to study the relationship between two proteins. For example, co-transduction of differently tagged proteins allows co-immunoprecipitation studies to be performed in almost any cell type. Therefore, we have designed our vector platform in such a way that cells transduced with two different constructs can readily be selected. Furthermore, the platform allows both proteins to be co-induced upon addition of Dox. To accomplish this we have chosen to replace the blast ${ }^{R}$ in our vectors containing a Tet-inducible promotor with a puro $^{R}$-IRES- rtTA3 sequence. The latter not only introduces the regulatory rtTA3 protein but also changes the selection marker from blast $^{R}$ to puro ${ }^{R}$. When pDG1iTag/Fluo-blast and pDG2iTag/Fluo-puro vectors are both transfected into one cell, the rtTA3 encoded on pDG2iTag/Fluo-puro will also act in trans on the TRE-CMV ${ }_{\text {min }}$ promoter from the pDG1iTag/Fluo-blast vector (Figure 4A). This validation was done in $\mathrm{HaCaT}$ cells co-transduced with pDG1iEcad-eGFP-V5blast and pDG2iLuc-Flag-puro (Figure 4, $B$ and C). Cells that had stably integrated both constructs were selected using both blasticidin and puromycin (Figure 4B). Tightly controlled Dox-induced co-expression of both fusion proteins was shown by anti-Flag and anti-GFP WB and eGFP-fluorescence (Figure 3B, lower panels, and Figure 4C).

The inducibility of the expression vector system was further analyzed in a timecourse kinetics experiment and by Dox titration (Figure 4, D and E). Time-dependent protein induction was studied in $\mathrm{HaCaT}$ cells transduced with our inducible vector pDG2iFlag-puro containing the RIPK4 gene (21). Expression of the Flag-tagged transgene could be detected by WB starting $3 \mathrm{~h}$ after treatment with $1 \mu \mathrm{g} / \mathrm{mL}$ Dox and reached maximal expression levels $\sim 9 \mathrm{~h}$ after Dox addition (Figure 4D). In addition, dosedependent transgene expression could be achieved by modulating the Dox concentration used to induce luciferase-Flag and E-cadherin-eGFP-V5 expression in co-transduced cells (Figure 4E).

We designed and constructed a novel lentiviral vector set that allows users to choose between different C-terminal fusion tags, constitutive as well as inducible expression, and two selectable markers. Lentiviral transduction in combination with the appropriate antibiotics selection resulted in close to $100 \%$ of transduced cells producing the protein of interest, as demonstrated by the expression of tdTomato-V5 and E-cadherineGFP-V5 (Figure 3B and Supplementary Figure S2B), histone2-eGFP (Figure 3C), and eGFP (Supplementary Figure S2A). We also showed that the these vectors modified from the original Invitrogen pLenti6 vector are functional and provided a proof-of-principle using transgene overexpression. Similar to other vector systems, our platform could potentially be used for the knock-down of genes using RNAi technology $(6,10)$. Since our system is derived from the Gatewaycompatible pLenti6 vector, which can be used in combination with the BLOCK-iT Pol II miR RNAi kit (Invitrogen), transfer of microRNAs to our vector platform is feasible. Similarly to other vector systems (6), our platform could potentially be used in vivo. A major advantage of our vector set over those previously described ones $(6-8,10)$ is the potential for co-expression of two different constructs. For example, our system allows co-transduction and selection of cells containing two inducible constructs, two constitutive constructs, or one constitutive and one inducible construct, whereas the previously described pINDUCER set (6) is limited to inducible expression. Furthermore, in the pINDUCER set, different selection markers are only present in miRNA and cDNA expression vectors, which means that selection of co-transduced cells is only possible when combining miRNA and cDNA expression vectors. An analogous versatile vector system that allows a combination of inducible and constitutive expression along with the choice of a wide variety of protein tags was described by Campeau et al. (7). However, that system uses a different approach than ours because the choice of the entry vector determines the tag fused to the protein of interest, which means that whenever a different fusion partner is required, a different entry vector must be constructed. Furthermore, when conditional expression is required, the system described by Campeau et al. requires the availability or generation of cell lines that express the TetR tetracycline transactivator protein to allow conditional expression. In our system however, all of these properties are determined by the choice of the destination vector. Consequently, our system is ideally suited for high-content screening because it is directly compatible with existing Gateway-based cDNA-libraries such as the Ultimate ORF clone collection (Thermo Fisher Scentific, Waltham, MA) and the human ORFeome v8.1 collection (BCCM/LMBP Plasmid Collection, Ghent, Belgium). In addition, our system allows one-step generation of inducible cell lines through destination vectors providing both the Tet-inducible promotor and the Tet-responsive transactivator gene on a single vector.

\section{Author contributions}

P.D.G. designed and cloned the vector set, performed experiments, and wrote the manuscript. I.K., K.L., and I.B. helped with the cloning; S.G., C.E., C.U., G.T., and K.L. tested vector performance. W.D.S. revised sequences and submitted GenBank records. S.L, P.V, J.H., and W.D. designed and supervised experiments and wrote the manuscript.

\section{Acknowledgments}

We thank Harald Braun for the mST2-mycHis expression plasmid. mST2-mycHis purifications were performed in the Protein Service Facility (PSF) of the Flanders Institute for Biotechnology (VIB). This research has been supported by VIB; European grants: FP6 Epistem LSHB-CT-2005-019067, FP7 TuMIC 2008-201662, and Euregional PACT II; Belgian grants: Interuniversity Attraction Poles, IAP 6/18 and IAP7; Stichting tegen Kanker 2010-162; Flemish grants: FWO-Vlaanderen, 1.5.058.12N, G.0544.11, G.0226.09, 1.5.169.08N, G.0A45.12N, G.0214.09N, G.0942.10N; Ghent University grants: MRP, GROUP-ID consortium, Concerted Research Actions (01G019A8-B8). P.V. holds a Methusalem grant (BOF09/01M00709) from the Flemish Government. P.D.G. obtained a PhD fellowship with the "Instituut voor Innovatie door Wetenschap en Technologie." G.T. obtained a PhD fellowship with the "Fonds voor Wetenschappelijk Onderzoek-Vlaanderen." S.L. is a postdoctoral fellow with the "Fonds voor Wetenschappelijk Onderzoek-Vlaanderen."

\section{Competing interests}

The authors declare no competing interests.

\section{References}

1. Naldini, L., U. Blomer, P. Gallay, D. Ory, R. Mulligan, F.H. Gage, I.M. Verma, and D. Trono. 1996. In vivo gene delivery and stable transduction of nondividing cells by a lentiviral vector. Science 272:263-267. 
2. Brummelkamp, T.R., R. Bernards, and R. Agami. 2002. A system for stable expression of short interfering rnas in mammalian cells. Science 296:550-553.

3. Bandaranayake, A.D., C. Correnti, B.Y. Ryu, M. Brault, R.K. Strong, and D.J. Rawlings. 2011. Daedalus: A robust, turnkey platform for rapid production of decigram quantities of active recombinant proteins in human cell lines using novel lentiviral vectors. Nucleic Acids Res. 39:e143.

4. Georgievska, B., D. Kirik, and A. Bjorklund. 2004. Overexpression of glial cell line-derived neurotrophic factor using a lentiviral vector induces time- and dose-dependent downregulation of tyrosine hydroxylase in the intact nigrostriatal dopamine system. J. Neurosci. 24:6437-6445.

5. Gossen, M. and H. Bujard. 1992. Tight control of gene expression in mammalian cells by tetracycline-responsive promoters. Proc. Natl. Acad. Sci. USA 89:5547-5551.

6. Meerbrey, K.L., G. Hu, J.D. Kessler, K. Roarty, M.Z. Li, J.E. Fang, J.I. Herschkowitz, A.E. Burrows, et al. 2011. The pinducer lentiviral toolkit for inducible rna interference in vitro and in vivo. Proc. Natl. Acad. Sci. USA 108:3665-3670.

7. Campeau, E., V.E. Ruhl, F. Rodier, C.L. Smith, B.L. Rahmberg, J.O. Fuss, J. Campisi, P. Yaswen, et al. 2009. A versatile viral system for expression and depletion of proteins in mammalian cells. PLoS ONE 4:e6529.

8. Stegmeier, F., G. Hu, R.J. Rickles, G.J. Hannon, and S.J. Elledge. 2005. A lentiviral micrornabased system for single-copy polymerase ii-regulated rna interference in mammalian cells. Proc. Natl. Acad. Sci. USA 102:13212-13217.
9. Dickins, R.A., M.T. Hemann, J.T. Zilfou, D.R. Simpson, I. Ibarra, G.J. Hannon, and S.W. Lowe. 2005. Probing tumor phenotypes using stable and regulated synthetic microrna precursors. Nat. Genet. 37:1289-1295.

10. Shin, K.J., E.A. Wall, J.R. Zavzavadjian, L.A. Santat, J. Liu, J.I. Hwang, R. Rebres, T. Roach, et al. 2006. A single lentiviral vector platform for microrna-based conditional rna interference and coordinated transgene expression. Proc. Natl. Acad. Sci. USA 103:13759-13764.

11. Boukamp, P., R.T. Petrussevska, D. Breitkreutz, J. Hornung, A. Markham, and N.E. Fusenig. 1988. Normal keratinization in a spontaneously immortalized aneuploid human keratinocyte cell line. J. Cell Biol. 106:761-771.

12. DuBridge, R.B., P. Tang, H.C. Hsia, P.M. Leong, J.H. Miller, and M.P. Calos. 1987. Analysis of mutation in human cells by using an epstein-barr virus shuttle system. Mol. Cell. Biol. 7:379-387.

13. Das, A.T., X. Zhou, M. Vink, B. Klaver, K. Verhoef, G. Marzio, and B. Berkhout. 2004. Viral evolution as a tool to improve the tetracycline-regulated gene expression system. J. Biol. Chem. 279:18776-18782.

14. Markusic, D., R. Oude-Elferink, A.T. Das, B. Berkhout, and J. Seppen. 2005. Comparison of single regulated lentiviral vectors with rtta expression driven by an autoregulatory loop or a constitutive promoter. Nucleic Acids Res. 33:e63.

15. de Marco, A., E. Deuerling, A. Mogk, T. Tomoyasu, and B. Bukau. 2007. Chaperonebased procedure to increase yields of soluble recombinant proteins produced in e. Coli. BMC Biotechnol. 7:32.

16. Dinnis, D.M. and D.C. James. 2005. Engineering mammalian cell factories for improved recombinant monoclonal antibody production: Lessons from nature? Biotechnol. Bioeng. 91:180-189.

17. Meylan, E., F. Martinon, M. Thome, M. Gschwendt, and J. Tschopp. 2002. Rip4 (dik/pkk), a novel member of the rip kinase family, activates nf-kappa $b$ and is processed during apoptosis. EMBO Rep. 3:1201-1208.

18. Moran, S.T., K. Haider, Y. Ow, P. Milton, L. Chen, and S. Pillai. 2003. Protein kinase c-associated kinase can activate nfkappab in both a kinasedependent and a kinase-independent manner. J. Biol. Chem. 278:21526-21533.

19. Niessen, C.M., D. Leckband, and A.S. Yap. 2011 Tissue organization by cadherin adhesion molecules: Dynamic molecular and cellular mechanisms of morphogenetic regulation. Physiol. Rev. 91:691-731.

20. Seibel, N.M., J. Eljouni, M.M. Nalaskowski, and W. Hampe. 2007. Nuclear localization of enhanced green fluorescent protein homomultimers. Anal. Biochem. 368:95-99.

21. De Groote, P., H.T. Tran, M. Fransen, G. Tanghe, C. Urwyler, B. De Craene, K. Leurs, B. Gilbert, et al. 2015. A novel ripk4-irf6 connection is required to prevent epithelial fusions characteristic for popliteal pterygium syndromes. Cell Death Differ. 22:1012-1024.

Received 17 July 2015; accepted 05 February 2016.

Address correspondence to Jurgen Haustraete or Wim Declercq, Inflammation Research Center, the Flanders InstituteforBiotechnology(VIB), 9052 Ghent, Belgium. E-mail: Wim.Declercq@irc.vib-ugent.be or Jurgen.Haustraete@irc.vib-ugent.be

To purchase reprints of this article, contact: biotechniques@fosterprinting.com

\section{RNAzol ${ }^{\circledR}$ RT Column Kit}

Universal RNA isolation kit from Molecular Research Center

The market leader providing the best RNA isolation methods for over 25 years

Fast and dependable isolation

Highest yield and purity

No DNase treatment necessary
Process small or large samples

Up to $\mathbf{3 0 0}$ mg RNA per column

Variety of solid or liquid samples

\section{Specialized protocols for the isolation of:}

Total RNA containing all cellular RNA Large RNA containing mRNA, rRNA and non-coding RNA Small RNA containing micro RNA

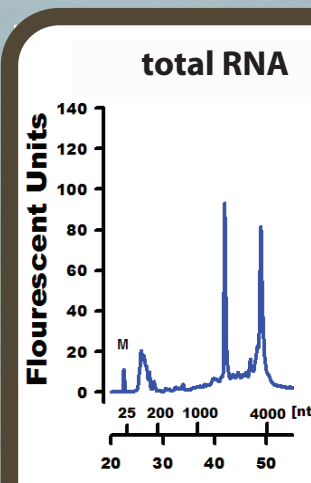

large RNA

small RNA

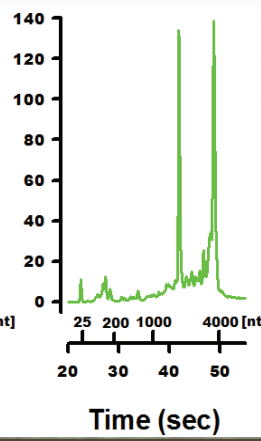

